University of Zurich

Department of Economics

Working Paper Series

ISSN 1664-7041 (print)

ISSN 1664-705X (online)

Working Paper No. 255

\title{
On Linear Transformations of Intersections
}

Alexey Kushnir and Shuo Liu

June 2017 


\title{
ON LinEAR TRANSFORMATIONS OF INTERSECTIONS
}

\author{
Alexey Kushnir and Shuo Liu*
}

June 3, 2017

\begin{abstract}
For any linear transformation and two convex closed sets, we provide necessary and sufficient conditions for when the transformation of the intersection of the sets coincides with the intersection of their images. We also identify analogous conditions for non-convex sets, general transformations, and multiple sets. We demonstrate the usefulness of our results via an application to the economics literature of mechanism design.
\end{abstract}

Keywords: linear transformation, convex closed set, intersection, directional convexity, mechanism design, dominant-strategy implementation, Bayesian implementation

\section{Introduction}

Let $T: \mathbb{R}^{n} \rightarrow \mathbb{R}^{m}$ be a linear transformation for some $m, n \in \mathbb{N}$ and $A, B \subset \mathbb{R}^{n}$ be two convex closed sets with a non-empty intersection. Generally, the transformation of the intersection $T(A \cap B)$ is a subset of the intersection of their images $T A \cap T B$. In this paper, we provide necessary and sufficient conditions for these sets to coincide, i.e., $T(A \cap B)=T A \cap T B$. To the best of our knowledge, this question has not been addressed previously.

Specifically, in Section 2, we provide conditions that exploit the directional convexity and pathconnectedness of the union $A \cup B$ and their relation to the kernel of the linear transformation. As shown in Section 3, these conditions can be extended to non-convex sets, non-linear transformations, and multiple sets. To further deepen our understanding of the problem, in Section 4, we

*Alexey Kushnir: Tepper School of Business, Carnegie Mellon University, Posner Hall 237, Pittsburgh, PA 15213, USA; Shuo Liu: Department of Economics, University of Zürich, Schönberggasse 1, CH-8001, Zürich, Switzerland. Email: alexey.kushnir@gmail.com and shuo.liu@econ.uzh.ch. We are very grateful to Itai Ashlagi, Egon Balas, Heinz Bauschke, Jérôme Bolte, Boris Bukh, Keenan Crane, Patrick Combettes, Gerard Cornuejols, Federico Echenique, Alfred Galichon, Ben Golub, Sergiu Hart, Fatma Kılınç-Karzan, Michael McCoy, Javier Pena, Marek Pycia, R Ravi, Stephen Spear, Rakesh Vohra, Josephine Yu, Weijie Zhong and seminar participants at Columbia University, Carnegie Mellon University, and the University of Pittsburgh for discussions and useful suggestions. 

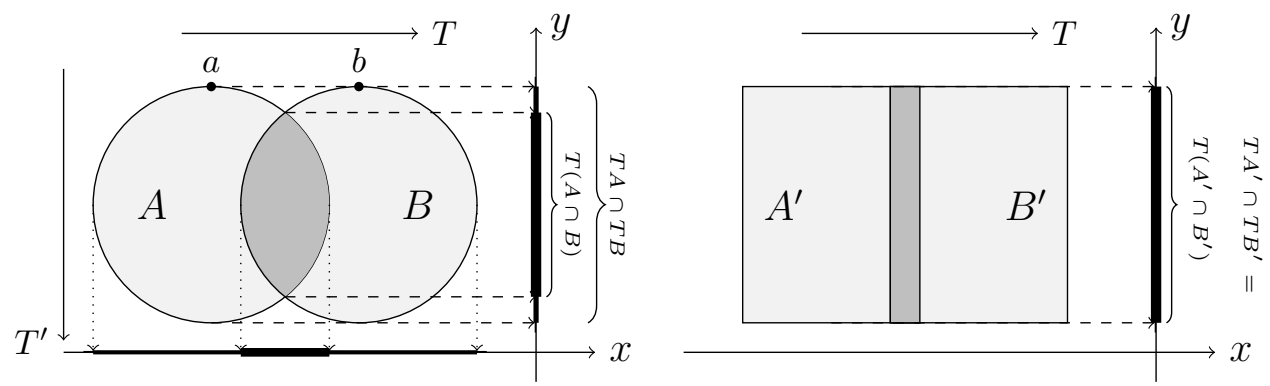

Figure 1. The orthogonal projection of $\operatorname{discs} A$ and $B$ (left) along the $x$-axis (transformation $T$ ) and the $y$-axis (transformation $\left.T^{\prime}\right)$. $T A \cap T B \neq T(A \cap B)$ and $T^{\prime} A \cap T^{\prime} B=T^{\prime}(A \cap B)$ because $A \cup B$ is not convex in the direction of the $x$-axis, but is convex in the direction of the $y$-axis. For rectangles $A^{\prime}$ and $B^{\prime}$ (right), $T A^{\prime} \cap T B^{\prime}=T\left(A^{\prime} \cap B^{\prime}\right)$ for any linear transformation $T$ because union $A^{\prime} \cup B^{\prime}$ is convex.

consider a different set of conditions that rely on the properties of support points of the convex sets. Finally, in Section 5, we demonstrate how our results can be applied to study the design of robust mechanisms for selling goods - a problem that has recently attracted attention in the economics literature of mechanism design (see, e.g., Manelli and Vincent, 2010; Gershkov, Goeree, Kushnir, Moldovanu and Shi, 2013).

\section{Convex Closed Sets}

To motivate, let us first consider a simple example. The left panel of Figure 1 presents two discs of the same radius $A, B \subset \mathbb{R}^{2}$ with non-empty intersection. If we project the discs along the $x$-axis, we obtain $T A \cap T B \neq T(A \cap B)$, where $T$ is the projection operator. The transformation of the intersection of the sets does not coincide with intersections of their images because there exist points $a \in A$ and $b \in B$ that have the same image, but the line connecting them contains no points of $A \cap B$. Note that such a situation is not possible when the union $A \cup B$ is convex, as the right panel of Figure 1 shows. This observation leads to our first result.

Theorem 1. For any linear transformation $T$ and convex closed sets $A$ and $B$, if the union $A \cup B$ is convex, then $T(A \cap B)=T A \cap T B$.

Proof. Consider any $A, B \subset \mathbb{R}^{n}$, linear $T: \mathbb{R}^{n} \rightarrow \mathbb{R}^{m}$, and $t \in T A \cap T B$. By definition, there exist $a \in A$ and $b \in B$ such that $T a=T b=t$. As $A \cup B$ is convex, we have $[a, b] \subset A \cup B$. Moreover, $[a, b] \cap A=\left[a, c_{1}\right]$ for some $c_{1} \in[a, b] \cap A$. Similarly, $[a, b] \cap B=\left[c_{2}, b\right]$ for some $c_{2} \in[a, b] \cap B$. As $\left[a, c_{1}\right] \cup\left[c_{2}, b\right]=[a, b]$, we have $\left[a, c_{1}\right] \cap\left[c_{2}, b\right] \neq \emptyset$, and thus there must exist some $c \in[a, b] \cap A \cap B$. By the linearity of $T$, we also have $t=T c \in T(A \cap B)$. Hence, $T(A \cap B)=T A \cap T B$. 
Remark. The convexity of the union of sets $A \cup B$ is also a necessary condition for $T(A \cap B)=$ $T A \cap T B$ for all linear transformations $T: \mathbb{R}^{n} \rightarrow \mathbb{R}^{n-1}$ (see Theorem A1 in the Appendix).

Theorem 1 provides a sufficient condition for $T(A \cap B)=T A \cap T B$ to be true for any linear transformation $T$. However, if only a specific transformation $T$ is of interest, this condition might be too demanding. For example, even though the union of the two discs in the left panel of Figure 1 is not convex, for projection $T^{\prime}$ along the $y$-axis, we nevertheless have $T^{\prime}(A \cap B)=T^{\prime} A \cap T^{\prime} B$. Intuitively, this is the case because there is no gap between sets $A$ and $B$ along the direction of the projection $T^{\prime}$. This motivates us to introduce the following concept of directional convexity 1

Definition 1. A set $C \subset \mathbb{R}^{n}$ is convex in direction $d \in \mathbb{R}^{n}$ if for all $a, b \in C$ with $a-b=\alpha d$ for some $\alpha \in \mathbb{R}$, we have $[a, b] \subset C$.

The left panel of Figure 1 shows that the union of discs $A$ and $B$ is convex in the direction parallel to the $y$-axis. Denoting the kernel of a linear transformation $T: \mathbb{R}^{n} \rightarrow \mathbb{R}^{m}$ as $\operatorname{ker}(T)=\left\{a \in \mathbb{R}^{n}: T a=0\right\}$, we establish the following result.

Theorem 2. For a linear transformation $T$ and convex closed sets $A$ and $B$, if the union $A \cup B$ is convex in every direction $d \in \operatorname{ker}(T)$, then $T(A \cap B)=T A \cap T B$.

Proof. The proof follows the steps of the one of Theorem 1. We omit it to avoid repetition.

Nevertheless, the convexity of the union with respect to the directions in the kernel is not generally a necessary condition. To illustrate, consider the two right triangular prisms $A$ and $B$ in Figure 2 and suppose we project them orthogonally along the $x y$-plane onto the $z$-axis. The projection of the intersection coincides with the intersection of the prisms' images. However, the union of the prisms is not convex with respect to the direction of the $x$-axis, which belongs to $\operatorname{ker}(T)$. To state our necessary condition, we introduce the following definition.

Definition 2. A set $C \subset \mathbb{R}^{n}$ is path-connected with respect to $\operatorname{ker}(T)$ of linear transformation $T: \mathbb{R}^{n} \rightarrow \mathbb{R}^{m}$ if the inverse image

$$
T_{C}^{-1}(t)=\{a \in C \mid a-b \in \operatorname{ker}(T) \text { for some } b: T b=t\}
$$

is path-connected for all $t \in T C$, where $T C$ refers to the image of $C$.

\footnotetext{
${ }^{1}$ Aumann and Hart (1986) previously introduced a similar concept of bi-convex sets that are convex with respect to two orthogonal directions.
} 

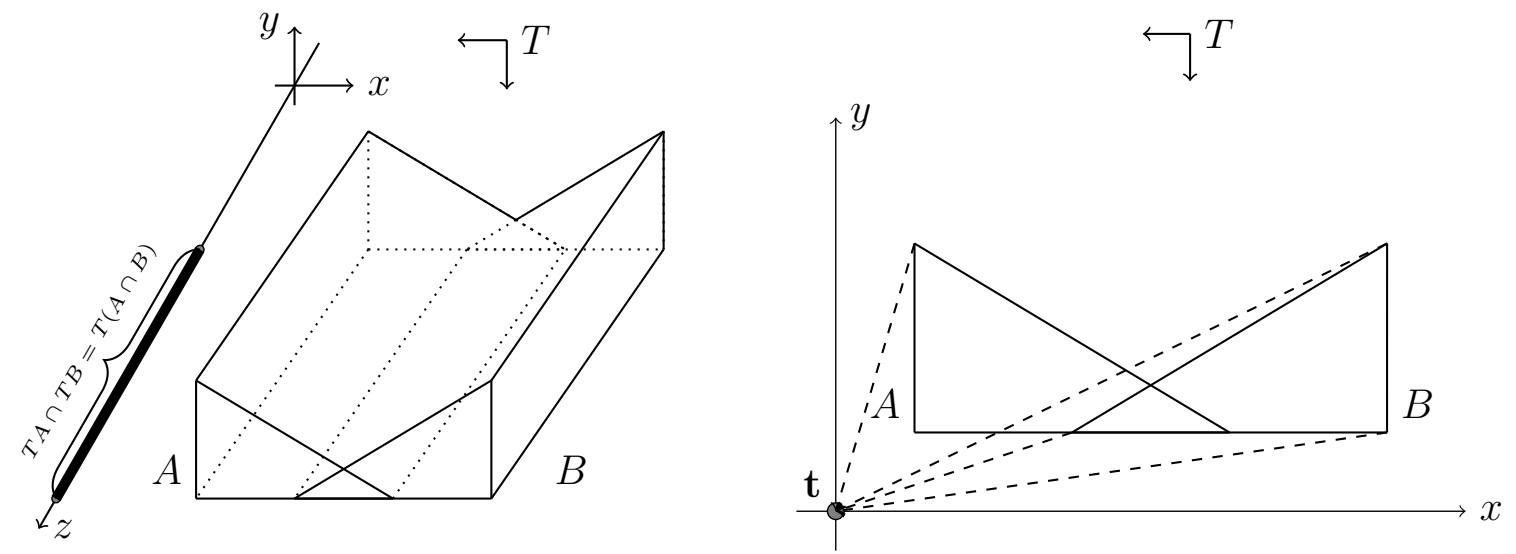

Figure 2. The left panel shows the orthogonal projection $T$ of prisms $A$ and $B$ along the $x y$-plane on the $z$-axis. The right shows the inverse image of a given point $t \in T A \cap T B$.

The right panel of Figure 2 presents the inverse image $T_{A \cup B}^{-1}(t)$ for some $t \in T A \cap T B$. Obviously, this is not a convex set. Nevertheless, this set is path-connected. It turns out that this is not a coincidence: That sets $T_{A \cup B}^{-1}(t)$ are path-connected for all $t \in T(A \cup B)$ is both necessary and sufficient for $T(A \cap B)=T A \cap T B$. This result is formally stated and proved in the next theorem.

Theorem 3. For a linear transformation $T$ and convex closed sets $A$ and $B$,

$$
T(A \cap B)=T A \cap T B
$$

if and only if $A \cup B$ is path-connected with respect to $\operatorname{ker}(T)$.

Proof. (If statement) Consider any $t \in T A \cap T B$. By definition, there exist $a \in A$ and $b \in B$ such that $T a=T b=t$. As $T_{A \cup B}^{-1}(t)$ is path-connected there exists a path $P(a, b) \subset T_{A \cup B}^{-1}(t)$ connecting $a$ and $b$. Moreover, $P(a, b) \cap A=P\left(a, c_{1}\right)$ is a path for some $c_{1} \in P(a, b) \cap A$. Similarly, $P(a, b) \cap B=P\left(c_{2}, b\right)$ is a path for some $c_{2} \in P(a, b) \cap B$. As $P\left(a, c_{1}\right) \cup P\left(c_{2}, b\right)=P(a, b)$, we must have $P\left(a, c_{1}\right) \cap P\left(c_{2}, b\right) \neq \emptyset$. This implies that there exists some $c \in P(a, b) \cap A \cap B$. Because $c \in T_{A \cup B}^{-1}(t)$, we also have $t=T c \in T(A \cap B)$. Hence, $T(A \cap B)=T A \cap T B$.

(Only-if statement) Consider any $a \in A \cup B$. Suppose first that $t=T a \notin T B$, and hence $T_{A \cup B}^{-1}(t)=T_{A}^{-1}(t)$. In this case, the inverse image $T_{A \cup B}^{-1}(t)$ is path-connected because set $A$ is convex and $T$ is linear. Similarly, if $T a \notin T A$, we have $T_{A \cup B}^{-1}(t)=T_{B}^{-1}(t)$, which is again pathconnected. Finally, suppose that $t=T a \in T A \cap T B$ and, without loss of generality, $a \in A$. As 
$A$ is convex and $T$ is linear, point $a$ is path-connected to any point in $T_{A \cup B}^{-1}(t) \cap A$. Let us now consider some $b \in T_{A \cup B}^{-1}(t) \cap B$. As $T(A \cap B)=T A \cap T B$, there exists $c \in A \cap B$ such that $t=T c$. As a result, $c \in A \cap B \cap T_{A \cup B}^{-1}(t)$. Therefore, point $c$ is path-connected with both $a$ and $b$ within $T_{A \cup B}^{-1}(t)$, which further implies that $a$ is also path-connected with $b$ within $T_{A \cup B}^{-1}(t)$.

Note that if $A \cup B$ is convex with respect to every direction in $\operatorname{ker}(T)$, the union is also pathconnected with respect to $\operatorname{ker}(T)$. In fact, in this case, any two points $a, b \in T_{A \cup B}^{-1}(t)$ are connected with a straight line. Theorem 3 , thus, weakens the sufficient condition of Theorem 2 by allowing points of the union to be connected with some path that is not necessarily a straight line.

\section{Extensions}

In this section, we show that the conditions set out in Section 2 can be extended to non-convex sets, general non-linear transformations, and multiple sets. We start by noting that the sufficiency part of Theorem 3 does not require sets $A$ and $B$ to be convex. Moreover, as the following result shows, the convexity assumption in the necessity part can be replaced with a weaker requirement whereby sets $A$ and $B$ are both path-connected with respect to $\operatorname{ker}(T)$.

Theorem 4 (Non-Convex Sets). Consider a linear transformation $T$ and closed sets $A$ and $B$ that are path-connected with respect to $\operatorname{ker}(T)$. Then,

$$
T(A \cap B)=T A \cap T B
$$

if and only if $A \cup B$ is path-connected with respect to $\operatorname{ker}(T)$.

Proof. The proof of the if statement repeats the steps of the sufficiency proof of Theorem 3. For the necessity part, the path-connectedness of $T_{A \cup B}^{-1}(t)$ for points $t \in T A \cap T B$ follows from Theorem 5 presented below. In regard to points $t \notin T A \cap T B$, the path-connectedness of $T_{A \cup B}^{-1}(t)$ follows from the assumption that both $A$ and $B$ are path-connected with respect to $\operatorname{ker}(T)$.

The linearity assumption of the transformation can also be dropped. However, for non-linear transformations $a-b \in \operatorname{ker}(T)$ does not imply $T a=T b$. To address this problem, we reformulate the notion of the inverse image as $T_{C}^{-1}(t)=\{a \in C \mid T a=t\}$. We can then modify the proof of Theorem 3 and obtain the following necessary and sufficient condition for general non-linear transformations. 
Theorem 5 (Non-Linear Transformations). Consider any transformation $T$ and closed sets $A$ and $B$ that have path-connected inverse images $T_{A}^{-1}(t)$ and $T_{B}^{-1}(t)$ for all $t \in T A \cap T B$. Then,

$$
T(A \cap B)=T A \cap T B
$$

if and only if the inverse image $T_{A \cup B}^{-1}(t)$ is path-connected for all $t \in T A \cap T B$.

Proof. The proof of the "if-statement" repeats the steps of the sufficiency proof of Theorem 3. Let us prove the necessity statement. Consider any $t \in T A \cap T B$. By definition, there exist $a \in T_{A}^{-1}(t)$ and $b \in T_{B}^{-1}(t)$. As $T(A \cap B)=T A \cap T B$, there also exists $c \in A \cap B$ such that $T c=t$. As $c \in T_{A}^{-1}(t)$ and $T_{A}^{-1}(t)$ is path-connected, there is a path in $T_{A}^{-1}(t)$ connecting $c$ and a. Similarly, $c \in T_{B}^{-1}(t)$ implies that there is also a path in $T_{B}^{-1}(t)$ connecting $c$ and $b$. But then, as $T_{A \cup B}^{-1}(t)=T_{A}^{-1}(t) \cup T_{B}^{-1}(t)$, there is a path in $T_{A \cup B}^{-1}(t)$ connecting $a$ and $b$.

Similar to the convexity assumption in Theorem 1, the inverse images $T_{A}^{-1}(t)$ and $T_{B}^{-1}(t)$ being path-connected for all $t \in T A \cap T B$ is only needed for the only-if statement. The sufficiency part does not require this additional condition.

The analysis of two convex closed sets can be readily extended to multiple sets. Let $A_{1}, \ldots, A_{J}$ be $J \geq 2$ convex closed sets. First, we present a direct generalization of Theorem 2, In particular, the theorem below states that if the union of every pair of sets is convex in every direction in the kernel, then the transformation of the intersection of all sets coincides with the intersection of their images.

Theorem 6 (Multiple Sets - Sufficiency). For a linear transformation $T: \mathbb{R}^{n} \rightarrow \mathbb{R}^{m}$ and $J$ convex closed sets $A_{1}, \ldots, A_{J}$,

$$
T\left(\cap_{j=1}^{J} A_{j}\right)=\cap_{j=1}^{J} T A_{j}
$$

if $A_{j} \cup A_{j^{\prime}}$ is convex in every direction $d \in \operatorname{ker}(T)$ for all $j, j^{\prime} \in \mathcal{J} \equiv\{1, \ldots, J\}$.

Proof. We prove the statement by induction. Consider some $t \in \cap_{j=1}^{J} T A_{j}$. By definition, there exists $x_{1} \in A_{1}$ such that $T x_{1}=t$. In addition, as $A_{1} \cup A_{2}$ is convex in every direction $d \in \operatorname{ker}(T)$, we know by Theorem 2 that there exists $x_{2} \in A_{1} \cap A_{2}$ such that $T x_{2}=t$. 
Now suppose that there exists $x_{j} \in A_{1} \cap \ldots \cap A_{j}$ such that $T x_{j}=t$. Consider some $y \in A_{j+1}$ such that $T y=t$, which again exists by definition. As $A_{i} \cup A_{j+1}$ is convex in every direction $d \in \operatorname{ker}(T)$, we have $\left[x_{j}, y\right] \subset A_{i} \cup A_{j+1}$ for each $i=1, \ldots, j$. Hence, $\left[x_{j}, y\right] \subset\left(\cap_{i=1}^{j} A_{i}\right) \cup A_{j+1}$. As $\cap_{i=1}^{j} A_{i}$ and $A_{j+1}$ are both convex and closed, we can conclude (similar to Theorem 1) that there must exist point $z \in\left(\cap_{i=1}^{j} A_{i}\right) \cap A_{j+1}$ such that $T z=t$.

Similar to Theorem 2, the condition of Theorem 6 is not generally necessary. Our necessary and sufficient condition for multiple sets is obtained by mirroring the proofs of Theorems 3 and 5 .

Theorem 7 (Multiple Sets - Characterization). For a linear transformation $T$ and $J$ convex closed sets $A_{1}, \ldots, A_{J}$,

$$
T\left(\cap_{j=1}^{J} A_{j}\right)=\cap_{j=1}^{J} T A_{j}
$$

if and only if $T_{\left(\cap_{i=1}^{j} A_{i}\right) \cup A_{j+1}}^{-1}(t)$ is path-connected for all $j=1, \ldots, J-1$ and for all $t \in \cap_{j=1}^{J} T\left(A_{j}\right)$.

Proof. (If statement) Without loss, let $J \geq 3$. Consider any $t \in \cap_{j=1}^{J} T A_{j}$. As $T_{A_{1} \cup A_{2}}^{-1}(t)$ is path-connected and $t \in T A_{1} \cap T A_{2}$, we have $t \in T\left(A_{1} \cap A_{2}\right)$. Hence, $t \in T\left(A_{1} \cap A_{2}\right) \cap T A_{3}$. Furthermore, as $T_{\left(A_{1} \cap A_{2}\right) \cup A_{3}}^{-1}(t)$ is also path-connected, we have $t \in T\left(A_{1} \cap A_{2} \cap A_{3}\right)$. Repeating this argument, we will finally have $t \in T\left(\cap_{j=1}^{J} A_{j}\right)$. Therefore, $T\left(\cap_{j=1}^{J} A_{j}\right)=\cap_{j=1}^{J} T A_{j}$.

(Only-if statement) Consider any $t \in \cap_{j=1}^{J} T A_{j}$ and any $j \in\{1, . ., J-1\}$. By linearity and convexity, the sets $T_{\cap_{i=1}^{j} A_{i}}^{-1}(t)$ and $T_{A_{j+1}}^{-1}(t)$ are both path-connected. Now consider any point $a \in T_{\cap_{i=1}^{j} A_{i}}^{-1}(t)$ and any point $b \in T_{A_{j+1}}^{-1}(t)$. As $\cap_{j=1}^{J} T A_{j}=T\left(\cap_{j=1}^{J} A_{j}\right)$, there must exist point $c \in \cap_{j=1}^{J=1} A_{j}$ such that $T c=t$. Because $c \in \cap_{i=1}^{j} A_{i}$ and $c \in A_{j+1}, c$ is path-connected to both $a$ and $b$ within $T_{\left(\cap_{j=1}^{k} A_{j}\right) \cup A_{k+1}}^{-1}(t)$. Therefore, $a$ and $b$ are also path-connected within $T_{\left(\cap_{j=1}^{k} A_{j}\right) \cup A_{k+1}}^{-1}(\mathrm{t})$.

\section{Dual Approach}

In this section, we examine our research question from a dual perspective. In particular, by exploiting the properties of the support points of the closed convex sets, we provide a different set of conditions for when a linear transformation of intersection coincides with the intersection of their images.

First, we introduce some useful notations. For a set $C \subset \mathbb{R}^{n}$, we define its support function as

$$
S^{C}(d)=\sup _{a \in C} a \cdot d
$$




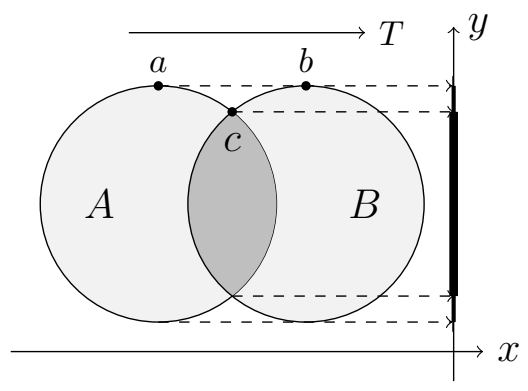

(a)

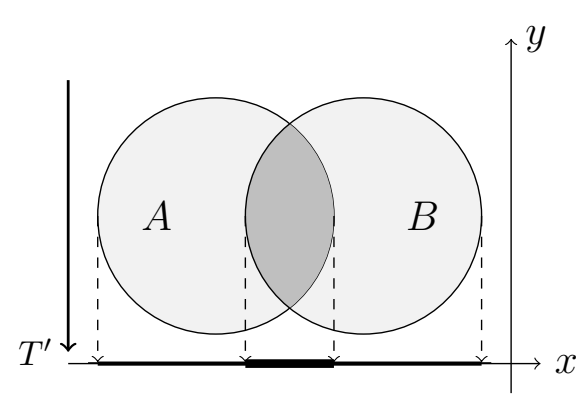

(b)

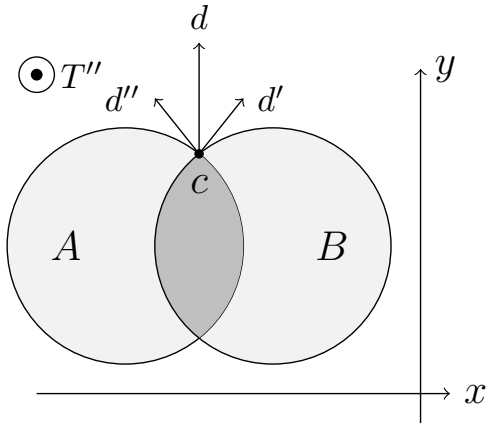

(c)

Figure 3. The orthogonal projections of $\operatorname{discs} A$ and $B$ along the $x$-axis (a), the $y$-axis (b), and the axis orthogonal to the $x y$-plane (c).

for all $d \in \mathbb{R}^{n}$. We refer to $u \in \mathbb{R}^{n}$ as a support point of set $C$ in direction $d \in \mathbb{R}^{n}$ if $u \in$ $\arg \max _{a \in C} a \cdot d$. The set of all support points of set $C$ in direction $d$ is denoted as $E(C, d)$. Note that $E(C, 0) \equiv C$ and that $E(C, d)$ is guaranteed to be non-empty if $C$ is compact. We also refer to the relative interior of set $C$ as ri $C !^{2}$ In addition, for any linear transformation $T: \mathbb{R}^{n} \rightarrow \mathbb{R}^{m}$, we denote by $\operatorname{ker}(T)^{\perp}=\left\{a \in \mathbb{R}^{n}: a \cdot d=0 \forall d \in \operatorname{ker}(T)\right\}$ the subspace orthogonal to $\operatorname{ker}(T)$. For simplicity, we assume that $n \geq m$ and $\operatorname{dim}(\operatorname{image}(T))=m$. This implies that $\operatorname{ker}(T)^{\perp}$ is a $m$-dimensional space.

To motivate the results in this section, consider a simple example as shown in Figure 3 . We consider again two discs $A$ and $B$ with a non-empty intersection that belongs to the $x y$-plane and their three orthogonal projections along the $x$-axis, the $y$-axis, and the axis orthogonal to the $x y$-plane. The orthogonal projection of $A \cap B$ along the $x$-axis does not coincide with the intersection of the images (Figure 3 a). Using the dual point of view, we can say that the problem arises because there exists a support point $c$ of $A \cap B$ that is neither a support point of $A$ nor a support point of $B$ in the direction $d$ orthogonal to the projection line, i.e., $d \in \operatorname{ker}(T)^{\perp}$. This implies that there must be distinct points $a$ and $b$ of sets $A$ and $B$ with a common image that lies above the image of point $c$.

However, this does not happen for projection $T^{\prime}$ along the $y$-axis (Figure $3 \mathrm{~b}$ ), where any support point of $A \cap B$ in the direction orthogonal to the projection line, i.e., $d \in \operatorname{ker}\left(T^{\prime}\right)^{\perp}$, is also a support point of one of the two sets in the same direction. This observation leads to our first sufficient condition in this section.

\footnotetext{
${ }^{2}$ Formally, the relative interior is defined by ri $C=\left\{a \in \operatorname{aff} C \mid \exists \varepsilon>0, B_{\varepsilon}(a) \cap\right.$ aff $\left.C \subset C\right\}$, where aff $C$ is the affine hull of set $C$ and $B_{\varepsilon}(a)$ is the Euclidean ball with radius $\varepsilon$ and center $a$ (see p. 44 in Rockafellar (1997)).
} 
Theorem 8. For a linear transformation $T$ and convex compact sets $A$ and $B$,

$$
\forall d \in \operatorname{ker}(T)^{\perp} E(A \cap B, d) \subseteq E(A, d) \cup E(B, d) \Longrightarrow T(A \cap B)=T A \cap T B .
$$

Proof. See the Appendix.

The condition of Theorem 8 , however, is not generally necessary. This can be readily illustrated with projection $T^{\prime \prime}$ of the two discs along the axis orthogonal to the $x y$-plane (Figure 3r). Though $T^{\prime \prime}(A \cap B)=T^{\prime \prime} A \cap T^{\prime \prime} B$, point $c$ is not a support point of either disc in the direction parallel to the $y$-axis, which belongs to $\operatorname{ker}\left(T^{\prime \prime}\right)^{\perp}$. Theorem 9 below shows that a necessary and sufficient condition requires any support point $c$ of $A \cap B$ in direction $d \in \operatorname{ker}\left(T^{\prime \prime}\right)^{\perp}$ to be a support point of each set in some directions $d^{\prime}, d^{\prime \prime} \in \operatorname{ker}\left(T^{\prime \prime}\right)^{\perp}$ with $d^{\prime}+d^{\prime \prime}=d$.

Theorem 9. For a linear transformation $T$ and convex compact sets $A$ and $B$ with $r i A \cap$ ri $B \neq \emptyset$,

$$
T(A \cap B)=T A \cap T B
$$

if and only if $\forall d \in \operatorname{ker}(T)^{\perp}$ and $\forall u \in E(A \cap B, d), \exists d^{\prime}, d^{\prime \prime} \in \operatorname{ker}(T)^{\perp}$ such that $d^{\prime}+d^{\prime \prime}=d$ and $u \in E\left(A, d^{\prime}\right) \cap E\left(B, d^{\prime \prime}\right)$.

Proof. See the Appendix.

While compact convex sets are fully characterized by their support points, for general closed convex sets, there might be directions without support points. Hence, to formulate a proper condition, we invoke the concept of the support function. As stated in the proof of Theorem 9, the support function for the intersection of sets $A \cap B$ with non-empty intersection of their relative interiors (i.e., ri $A \cap$ ri $B \neq \emptyset$ ) can be conveniently characterized by ${ }^{3}$

$$
S^{A \cap B}(d)=\inf _{\substack{d^{\prime}+d^{\prime \prime}=d \\ d^{\prime}, d^{\prime \prime} \in \mathbb{R}^{n}}}\left(S^{A}\left(d^{\prime}\right)+S^{B}\left(d^{\prime \prime}\right)\right) .
$$

Our last necessary and sufficient condition states that for every direction $d \in \operatorname{ker}(T)^{\perp}$, we can limit ourselves in the above equation to a minimization among only directions $d^{\prime}, d^{\prime \prime} \in \operatorname{ker}(T)^{\perp}$.

${ }^{3}$ The condition on relative interiors can be relaxed. In that case, for any $d \in \mathbb{R}^{n}$, we have

$$
S^{A \cap B}(d)=\operatorname{cl}\left(\inf _{\substack{d^{\prime}+d^{\prime \prime}=d \\ d^{\prime}, d^{\prime \prime} \in \mathbb{R}^{n}}}\left(S^{A}\left(d^{\prime}\right)+S^{B}\left(d^{\prime}\right)\right)\right),
$$

where $c l f$ refers to the closure of $f$, i.e., $c l f(d)=\liminf _{d^{\prime} \rightarrow d} f\left(d^{\prime}\right)$ (p. 78 in Hiriart-Urrut and Lemaréchal (2012)). 
Theorem 10. For a given transformation $T$ and convex closed sets $A$ and $B$ with ri $A \cap$ ri $B \neq \emptyset$, $T(A \cap B)=T A \cap T B$ if and only if for any direction $d \in \operatorname{ker}(T)^{\perp}$

$$
\inf _{\substack{d^{\prime}+d^{\prime \prime}=d \\ d^{\prime}, d^{\prime \prime} \in \mathbb{R}^{n}}}\left(S^{A}\left(d^{\prime}\right)+S^{B}\left(d^{\prime \prime}\right)\right)=\inf _{\substack{d^{\prime}+d^{\prime \prime}=d \\ d^{\prime}, d^{\prime \prime} \in \operatorname{ker}(T)^{\perp}}}\left(S^{A}\left(d^{\prime}\right)+S^{B}\left(d^{\prime}\right)\right) .
$$

Proof. See the Appendix.

Though the above result seems to be rather technical, it proves to be very useful in the application considered in the following section.

\section{Application}

We now present an economic application of our main results to designing robust mechanisms for selling goods. To keep the exposition clear and transparent, we study only a simple example. Consider an auctioneer who wants to sell an object to two agents indexed by $i=1,2$. Agent $i$ 's valuation of the object $x^{i}$ can be one of two types, $x_{l}$ or $x_{h}$, and it is independently distributed with equal probability. In addition, if agent $i$ pays $p^{i}$ to obtain the object his utility equals $x^{i}-p^{i}$.

Using the revelation principle (Myerson, 1979), we restrict our analysis to direct mechanisms, where agents are asked to report their types. Specifically, each mechanism specifies the probabilities that the object is allocated to agents and the payments requested from agents conditional on their reported types. To predict the outcome of a mechanism, economists typically consider two solution concepts. The first solution concept is Bayesian incentive compatibility (BIC), which requires that each agent reports his type truthfully given his belief about the distribution of the other agent types and that the other agents also report their types truthfully. The second solution concept is dominant-strategy incentive compatibility (DIC), which requires that each agent reports his type truthfully regardless of the other agents' reports and the realizations of their types ${ }^{4} \mathrm{We}$ apply the result of Theorem 10 to establish the following BIC-DIC equivalence result: For any BIC mechanism one could always construct an equivalent DIC mechanism that delivers the same interim allocation probabilities to all agents: ${ }^{5}$

\footnotetext{
${ }^{4} \mathrm{DIC}$ is a rather demanding solution concept that guarantees good and robust behavior of agents in applications. In particular, it does not require that agents have a correct belief regarding the distribution of others' types, and it permits mistakes in agents' behavior. DIC mechanisms have been successfully adopted by many markets ranging from spectrum auctions (Milgrom, 2002) to labor markets for doctors, kidney exchange, and school choice in major US cities (Roth, 2008).

${ }^{5}$ Manelli and Vincent (2010) were the first to establish this result and Gershkov et al. (2013) extended it
} 
To start with, note that any allocation of the object can be summarized by vectors $q^{i}=$ $\left(q_{l l}^{i}, q_{l h}^{i}, q_{h l}^{i}, q_{h h}^{i}\right), i=1,2$, where each element refers to the probability that agent $i$ receives the object and the first (second) subscript refers to the first (second) agent's type. From agent 1's point of view, the expected probability of receiving an object equals $Q_{l}^{1}=\frac{1}{2} q_{l l}^{1}+\frac{1}{2} q_{l h}^{1}$ and $Q_{h}^{1}=\frac{1}{2} q_{h l}^{1}+\frac{1}{2} q_{h h}^{1}$, and similarly for agent 2 . We refer to vectors $Q^{i}=\left(Q_{l}^{i}, Q_{h}^{i}\right), i=1,2$ as interim allocation probabilities.

Two types of constraints shape the set of available allocation rules: feasibility constratings and incentive compatibility constraints. Feasibility constraints guarantee that the object is not allocated to two agents simultaneously.

$$
\left\{\begin{array}{l}
q_{l l}^{1}+q_{l l}^{2} \leq 1 \\
q_{l l}^{1}, q_{l l}^{2} \geq 0
\end{array}, \quad\left\{\begin{array}{l}
q_{l h}^{1}+q_{l h}^{2} \leq 1 \\
q_{l h}^{1}, q_{l h}^{2} \geq 0
\end{array}, \quad\left\{\begin{array}{l}
q_{h l}^{1}+q_{h l}^{2} \leq 1 \\
q_{h l}^{1}, q_{h l}^{2} \geq 0
\end{array}, \quad\left\{\begin{array}{l}
q_{h h}^{1}+q_{h h}^{2} \leq 1 \\
q_{h h}^{1}, q_{h h}^{2} \geq 0
\end{array}\right.\right.\right.\right.
$$

Plainly, the feasibility set $C_{F} \subset \mathbb{R}^{8}$ defined by constraints $(3)$ consists of a Cartesian product of four simplices. The support function for each simplex is easy to derive, e.g., $\max \left(0, d_{l l}^{1}, d_{l l}^{2}\right)$ is the support function of the first simplex. The support function of a Cartesian product of sets equals the sum of the support function. Hence, the support function of $C_{F}$ for any $d \in \mathbb{R}^{8}$ equals

$$
S^{C_{F}}(d)=\max \left(0, d_{l l}^{1}, d_{l l}^{2}\right)+\max \left(0, d_{l h}^{1}, d_{l h}^{2}\right)+\max \left(0, d_{h l}^{1}, d_{h l}^{2}\right)+\max \left(0, d_{h h}^{1}, d_{h h}^{2}\right)
$$

Dominant strategy and Bayesian incentive compatibility constraints ensure that it is in the best interest of each agent to report his type truthfully. Laffont and Maskin (1980) showed that for any non-decreasing (ex post) allocation probabilities

$$
\left\{\begin{array}{l}
q_{l l}^{1} \leq q_{h l}^{1} \\
q_{l h}^{1} \leq q_{h h}^{1}
\end{array}, \quad\left\{\begin{array}{l}
q_{l l}^{2} \leq q_{l h}^{2} \\
q_{h l}^{2} \leq q_{h h}^{2}
\end{array}\right.\right.
$$

it is possible to find payments that jointly form a DIC mechanism. In addition, they showed that the above condition is also necessary for an allocation to be part of a DIC mechanism. We denote the above set of allocations as $C_{D I C}$ and its support function as $S^{D I C}$. Similarly, BIC constraints reduce to the monotonicity of interim allocation probabilities

$$
Q_{l}^{1} \leq Q_{h}^{1}, \quad Q_{l}^{2} \leq Q_{h}^{2}
$$

to social choice settings. Our treatment is, however, different from theirs, as it relies on the calculus of support functions. See Goeree and Kushnir (2017) for a fully developed approach to mechanism design that uses the calculus of support functions. 
Let $T: \mathbb{R}^{8} \rightarrow \mathbb{R}^{4}$ be the linear operator that transforms ex post allocations $\left(q^{1}, q^{2}\right)$ into interim allocations $\left(Q^{1}, Q^{2}\right)$. Under transformation $T$, the DIC constraints (5) map onto the BIC constraints (6) and the feasibility constraints (3) onto the corresponding feasibility constraints at the interim level. The question of the BIC-DIC equivalence then reduces to the question of whether the transformation of the intersection $T\left(C_{F} \cap C_{D I C}\right)$ coincides with $T C_{F} \cap T C_{D I C}$. We apply Theorem 10 to answer this question.

To this end, we first consider support function $S^{C_{F} \cap D I C^{1}}$ corresponding to the intersection of $C_{F}$ with the DIC constraints of only agent 1. The support function for the DIC constraints of agent 1 equals

$$
S^{D I C^{1}}(d)= \begin{cases}0 & \text { if } d=\lambda_{l}^{1}(1,0,-1,0,0,0,0,0)+\lambda_{h}^{1}(0,1,0,-1,0,0,0,0) \\ +\infty & \text { otherwise }\end{cases}
$$

for any $\lambda_{l}^{1}, \lambda_{h}^{2} \geq 0$. Given the result of Theorem 10, we need to analyze the values of $S^{C_{F} \cap D I C^{1}}(d)$ only for directions $d \in \operatorname{ker}(T)^{\perp}$. Any such direction can be described by an 8-dimensional vector $d=\left(d_{l}^{1}, d_{l}^{1}, d_{h}^{1}, d_{h}^{1}, d_{l}^{2}, d_{h}^{2}, d_{l}^{2}, d_{h}^{2}\right)$. Taking into account that the support function of the intersection equals the convolution of the corresponding support functions (see equation (1D), we obtain

$$
\begin{array}{r}
S^{C_{F} \cap D I C^{1}}(d)=\inf _{\lambda_{l}^{1}, \lambda_{h}^{1} \geq 0} \max \left(0, d_{l}^{1}-\lambda_{l}^{1}, d_{l}^{2}\right)+\max \left(0, d_{l}^{1}-\lambda_{h}^{1}, d_{h}^{2}\right)+ \\
\max \left(0, d_{h}^{1}+\lambda_{l}^{1}, d_{l}^{2}\right)+\max \left(0, d_{h}^{1}+\lambda_{h}^{1}, d_{h}^{2}\right) .
\end{array}
$$

The above minimization problem has a solution at $\lambda^{1}=\lambda_{l}^{1}=\lambda_{h}^{1}=\max \left(0, \frac{1}{2}\left(d_{l}^{1}-d_{h}^{1}\right)\right) \cdot{ }^{6}$ Denote $\tilde{d}_{l}^{1}=d_{l}^{1}-\lambda^{1}$ and $\tilde{d}_{h}^{1}=d_{h}^{1}+\lambda^{1}$. Introducing the DIC constraints for agent 2 , we similarly obtain

$$
\begin{array}{r}
S^{C_{F} \cap D I C^{1} \cap D I C^{2}}(d)=\inf _{\lambda_{l}^{2}, \lambda_{h}^{2} \geq 0} \\
\max \left(0, \tilde{d}_{l}^{1}, d_{l}^{2}-\lambda_{l}^{2}\right)+\max \left(0, \tilde{d}_{l}^{1}, d_{h}^{2}+\lambda_{l}^{2}\right)+ \\
\max \left(0, \tilde{d}_{h}^{1}, d_{l}^{2}-\lambda_{h}^{2}\right)+\max \left(0, \tilde{d}_{h}^{1}, d_{h}^{2}+\lambda_{h}^{2}\right) .
\end{array}
$$

The above minimization problem has again a solution at $\lambda^{2}=\lambda_{l}^{2}=\lambda_{h}^{2}=\max \left(0, \frac{1}{2}\left(d_{l}^{2}-d_{h}^{2}\right)\right)$. Overall, for all $d \in \operatorname{ker}(T)^{\perp}$, there exists a vector $\lambda=\left(\lambda^{1}, \lambda^{1},-\lambda^{1},-\lambda^{1}, \lambda^{2},-\lambda^{2}, \lambda^{2},-\lambda^{2}\right) \in \operatorname{ker}(T)^{\perp}$ such that $S^{C_{F} \cap D I C}(d)=S^{C_{F}}(d-\lambda)+S^{D I C}(\lambda)$. Hence, the condition of Theorem 10 is satisfied and we have $T\left(C_{F} \cap C_{D I C}\right)=T C_{F} \cap T C_{D I C}$, which establishes the BIC-DIC equivalence result.

The purpose of the above example is to illustrate the use of our results rather than to derive

\footnotetext{
${ }^{6}$ The fact that the minimum is achieved at the same values of $\lambda$ s has a deeper reason connecting the above minimization problem to majorization. See Goeree and Kushnir (2017) for a more detailed analysis.
} 
novel insights in the economics literature. A detailed analysis of the BIC-DIC equivalence in linear social choice problems with independent one-dimensional types using techniques from convex analysis has been provided by Goeree and Kushnir (2017). The results of the present study, however, apply beyond linear settings and models with one-dimensional types. As such our result offer a new and productive way to study the BIC-DIC equivalence in much more general environments, which, however, is a topic for future research.

\section{Conclusion}

In this paper, we studied when a transformation of the intersection of two closed sets coincides with the intersection of their images. Using both primal and dual approaches, we provided necessary and sufficient conditions for any linear transformation and any two convex closed sets. We also identified analogous conditions for non-convex sets, general transformations, and multiple sets.

As an application, we showed how our results can be applied to designing robust mechanisms for selling goods. In fact, our results can be used to compare not only incentive compatibility constraints but also any interim and ex post constraints, including individual rationality, budget balance. We consider this to be an exciting direction for future research. We also believe our results will be of great use by research in the fields of operations research, convex analysis, and computer science. 


\section{Appendix}

Theorem A1. Consider two convex closed sets $A, B \subset \mathbb{R}^{n}$. If for all linear transformations $T: \mathbb{R}^{n} \rightarrow \mathbb{R}^{n-1}$ we have $T(A \cap B)=T A \cap T B$, then $A \cup B$ is convex.

Proof. Take some $a \in A$ and $b \in B$ such that $a \neq b$, and consider a surjective linear transformation $T: \mathbb{R}^{n} \rightarrow \mathbb{V}$, where $\mathbb{V}=\left\{v \in \mathbb{R}^{n}: v \cdot(x-y)=0\right\}$ is $(n-1)$-dimensional. By construction, we have $a-b \in \operatorname{ker}(T)$ and $T a=T b=t$ for some $t \in \mathbb{R}^{n-1}$. As $t \in T A \cap T B=T(A \cap B)$, there exists $c \in A \cap B$ such that $T c=t$. As $\operatorname{dim}(\operatorname{ker}(T))=n-\operatorname{dim}(\mathbb{V})=1$, points $a, b, c$ must lie on one straight line. As $A$ and $B$ are convex and $c \in A \cap B$, we have $[a, c] \subset A$ and $[b, c] \subset B$, which implies that $[a, b] \subset A \cup B$. Hence, $A \cup B$ is convex.

Proof of Theorem 8. As $T(A \cap B) \subset T A \cap T B$ and both $T(A \cap B)$ and $T A \cap T B$ are convex compact sets, it suffices to show that $E\left(T(A \cap B), d_{m}\right) \subset E\left(T A \cap T B, d_{m}\right)$ for all directions $d_{m} \in \mathbb{R}^{m}$, that is, every support point of $T(A \cap B)$ is also a support point of $T A \cap T B$ in the same direction. For this purpose, consider any direction $d_{m} \in \mathbb{R}^{m}$. Let $T^{*}: \mathbb{R}^{m} \rightarrow \mathbb{R}^{n}$ be the adjoint operator (or the transpose) of $T$. As image $\left(T^{*}\right)=\operatorname{ker}(T)^{\perp}$ (see p. 120 in Axler (1997)) we have $T^{*}\left(d_{m}\right) \in \operatorname{ker}(T)^{\perp}$. The condition of the theorem then ensures that $E(A \cap$ $\left.B, T^{*}\left(d_{m}\right)\right) \subseteq E\left(A, T^{*}\left(d_{m}\right)\right) \cup E\left(B, T^{*}\left(d_{m}\right)\right)$. By the definition of adjoint operators, we further have $E\left(T(A \cap B), d_{m}\right) \subseteq E\left(T A, d_{m}\right) \cup E\left(T B, d_{m}\right)$.

Now consider any $t \in E\left(T(A \cap B), d_{m}\right) \subseteq E\left(T A, d_{m}\right) \cup E\left(T B, d_{m}\right)$. Without loss, we suppose that $t \in E\left(T A, d_{m}\right)$. On the one hand, $T(A \cap B) \subset T A \cap T B$ implies that $t \cdot d_{m} \leq t^{\prime} \cdot d_{m}$ for all $t^{\prime} \in E\left(T A \cap T B, d_{m}\right)$. On the other hand, as $T A \cap T B \subset T A$, it must be the case that $t \cdot d_{m} \geq t^{\prime} \cdot d_{m}$ for all $t^{\prime} \in E\left(T A \cap T B, d_{m}\right)$. Hence, overall we have $t \cdot d_{m}=t^{\prime} \cdot d_{m}$ for all $t^{\prime} \in E\left(T A \cap T B, d_{m}\right)$. As $t \in T A \cap T B$, we can conclude that $t \in E\left(T A \cap T B, d_{m}\right)$.

Proof of Theorem 9. For arbitrary direction $d_{m} \in \mathbb{R}^{m}$ the support function of the image of the intersection equals

$$
S^{T(A \cap B)}\left(d_{m}\right)=\sup _{t \in T(A \cap B)} t \cdot d_{m}=\sup _{x \in A \cap B} x \cdot T^{*}\left(d_{m}\right)=S^{A \cap B}\left(T^{*}\left(d_{m}\right)\right),
$$

where $T^{*}$ is the adjoint operator. As image $\left(T^{*}\right)=\operatorname{ker}(T)^{\perp}$ we have $T^{*}\left(d_{m}\right) \in \operatorname{ker}(T)^{\perp}$. The support function for the intersection of sets having non-empty intersection of their relative interiors ri $A \cap$ ri $B \neq \emptyset$ can be conveniently characterized by (see p. 145 in Rockafellar (1997))

$$
S^{A \cap B}\left(T^{*}\left(d_{m}\right)\right)=\inf _{\substack{d^{\prime}+d^{\prime \prime}=T^{*}\left(d_{m}\right) \\ d^{\prime}, d^{\prime \prime} \in \mathbb{R}^{n}}}\left(S^{A}\left(d^{\prime}\right)+S^{B}\left(d^{\prime \prime}\right)\right) .
$$


Taking into account the condition of the theorem and that the set of support points is non-empty in any direction for compact sets we obtain

$$
S^{A \cap B}\left(T^{*}\left(d_{m}\right)\right)=\inf _{\substack{d^{\prime}+d^{\prime \prime}=T^{*}\left(d_{m}\right) \\ d^{\prime}, d^{\prime \prime} \in \mathbb{R}^{n}}}\left(S^{A}\left(d^{\prime}\right)+S^{B}\left(d^{\prime \prime}\right)\right)=\inf _{\substack{d^{\prime}+d^{\prime \prime}=T^{*}\left(d_{m}\right) \\ d^{\prime}, d^{\prime \prime} \in \operatorname{ker}(T)^{\perp}}}\left(S^{A}\left(d^{\prime}\right)+S^{B}\left(d^{\prime \prime}\right)\right)
$$

As $d^{\prime}, d^{\prime \prime} \in \operatorname{ker}(T)^{\perp}=\operatorname{image}\left(T^{*}\right)$ there must exist $d_{m}^{\prime}, d_{m}^{\prime \prime} \in \mathbb{R}^{m}$ such that $d^{\prime}=T^{*}\left(d_{m}^{\prime}\right)$, and $d^{\prime \prime}=T^{*}\left(d_{m}^{\prime \prime}\right)$. Moreover, we must have $d_{m}^{\prime}+d_{m}^{\prime \prime}=d_{m} !^{7}$ Hence,

$$
\begin{aligned}
S^{A \cap B}\left(T^{*}\left(d_{m}\right)\right) & =\inf _{\substack{d_{m}^{\prime}+d_{m}^{\prime \prime}=d_{m} \\
d_{m}^{\prime}, d_{m}^{\prime \prime} \in \mathbb{R}^{m}}}\left(S^{A}\left(T^{*}\left(d_{m}^{\prime}\right)\right)+S^{B}\left(T^{*}\left(d_{m}^{\prime \prime}\right)\right)\right) \\
& =\inf _{\substack{d_{m}^{\prime}+d_{m}^{\prime \prime}=d_{m} \\
d_{m}^{\prime}, d_{m}^{\prime \prime} \in \mathbb{R}^{m}}}\left(S^{T A}\left(d_{m}^{\prime}\right)+S^{T B}\left(d_{m}^{\prime \prime}\right)\right)=S^{T A \cap T B}\left(d_{m}\right)
\end{aligned}
$$

Overall, $S^{T(A \cap B)}\left(d_{m}\right)=S^{T A \cap T B}\left(d_{m}\right)$ for all directions $d_{m} \in \mathbb{R}^{m}$. Hence, $T(A \cap B)=T A \cap T B$.

(Only-if statement) To establish the necessity part, we assume that $T(A \cap B)=T A \cap T B$ and consider any direction $d \in \operatorname{ker}(T)^{\perp}$ and support point $u \in E(A \cap B, d)$. As $\operatorname{ker}(T)^{\perp}=\operatorname{image}\left(T^{*}\right)$ there must exist $d_{m} \in \mathbb{R}^{m}$ such that $d=T^{*}\left(d_{m}\right)$. For this direction, we have

$$
S^{T A \cap T B}\left(d^{m}\right)=\inf _{\substack{d_{m}^{\prime}+d_{m}^{\prime \prime}=d_{m} \\ d_{m}^{\prime}, d_{m}^{\prime \prime} \in \mathbb{R}^{m}}}\left(S^{T A}\left(d_{m}^{\prime}\right)+S^{T B}\left(d_{m}^{\prime \prime}\right)\right)=\inf _{\substack{d_{m}^{\prime}+d_{m}^{\prime \prime}=d_{m} \\ d_{m}^{\prime}, d_{m}^{\prime \prime} \in \mathbb{R}^{m}}}\left(S^{A}\left(T^{*}\left(d_{m}^{\prime}\right)\right)+S^{B}\left(T^{*}\left(d_{m}^{\prime \prime}\right)\right)\right)
$$

For any $d_{m}^{\prime}, d_{m}^{\prime \prime}$ such that $d_{m}^{\prime}+d_{m}^{\prime \prime}=d_{m}$, let $d^{\prime}, d^{\prime \prime} \in \operatorname{ker}(T)^{\perp}$ and $d^{\prime}=T^{*}\left(d_{m}^{\prime}\right), d^{\prime \prime}=T^{*}\left(d_{m}^{\prime \prime}\right)$, $d^{\prime}+d^{\prime \prime}=T^{*}\left(d_{m}\right)=d$. As $T(A \cap B)=T A \cap T B$ there must exist $d^{\prime}$ and $d^{\prime \prime}$ such that

$$
u \cdot d=S^{A \cap B}\left(T^{*}\left(d_{m}\right)\right)=S^{T(A \cap B)}\left(d_{m}\right)=S^{T A \cap T B}\left(d_{m}\right)=S^{A}\left(d^{\prime}\right)+S^{B}\left(d^{\prime \prime}\right)
$$

where the last equality follows from Theorem 16.4 in Rockafellar (1997), which asserts that the infimum of $\mathrm{A} .3 \mathrm{n}$ is achieved when the relative interiors of the two sets have a point in common. Also, as $u \in A \cap B$, we have $u \cdot d^{\prime} \leq S^{A}\left(d^{\prime}\right)$ and $u \cdot d^{\prime \prime} \leq S^{B}\left(d^{\prime \prime}\right)$. As a result, we must have $u \cdot d^{\prime}=S^{A}\left(d^{\prime}\right)$, and $u \cdot d^{\prime \prime}=S^{B}\left(d^{\prime \prime}\right)$. In other words, $u \in E\left(A, d^{\prime}\right) \cap E\left(B, d^{\prime \prime}\right)$, where $d^{\prime}, d^{\prime \prime} \in \operatorname{ker}(T)^{\perp}$ and $d^{\prime}+d^{\prime \prime}=T^{*}\left(d_{m}\right)$. As the choice of $d_{m}$ is arbitrary and image $\left(T^{*}\right)=\operatorname{ker}(T)^{\perp}$ the only-if statement follows.

\footnotetext{
${ }^{7}$ To see that $d_{m}^{\prime}+d_{m}^{\prime \prime} \neq d_{m}$ is not possible, denote $a_{m}=d_{m}^{\prime}+d_{m}^{\prime \prime}-d_{m} \in$ image $(T)$. By construction, $T^{*}\left(a_{m}\right)=0$, and thus $a_{m} \in \operatorname{ker}\left(T^{*}\right)=\operatorname{image}(T)^{\perp}$. Hence, we have $a_{m} \in \operatorname{image}(T) \cap \operatorname{image}(T)^{\perp}$, which implies $a_{m}=0$.
} 
Proof of Theorem 10. The proof of the sufficient part follows from the proof of the sufficient part of Theorem 9. Let us now establish the necessary part. Assume that $T(A \cap B)=T A \cap T B$. This implies that for any $d_{m} \in \mathbb{R}^{m}$ we have $S^{T(A \cap B)}\left(d_{m}\right)=S^{T A \cap T B}\left(d_{m}\right)$. We know that

$$
\begin{aligned}
S^{T A \cap T B}\left(d_{m}\right) & =\inf _{\substack{d^{\prime}+d^{\prime \prime}=d_{m} \\
d_{m}^{\prime}, d_{m}^{\prime \prime} \in \mathbb{R}^{m}}}\left(S^{T A}\left(d_{m}^{\prime}\right)+S^{T} B\left(d_{m}^{\prime \prime}\right)\right) \\
& =\inf _{\substack{d_{m}^{\prime}+d_{m}^{\prime \prime}=d_{m} \\
d_{m}^{\prime}, d_{m}^{\prime \prime} \in \mathbb{R}^{m}}}\left(S^{A}\left(T^{*} d_{m}^{\prime}\right)+S^{B}\left(T^{*} d_{m}^{\prime \prime}\right)\right) .
\end{aligned}
$$

As image $\left(T^{*}\right)=\operatorname{ker}(T)^{\perp}$, there must exist $d^{\prime}, d^{\prime \prime} \in \operatorname{ker}(T)^{\perp}$ such that $d^{\prime}=T^{*} d_{m}^{\prime}, d^{\prime \prime}=T^{*} d_{m}^{\prime \prime}$. Similar to the proof of Theorem 9 (see footnote 3 ) we establish that sets $\left\{d^{\prime}, d^{\prime \prime} \in \operatorname{ker}(T)^{\perp} \mid d^{\prime}+d^{\prime \prime}=\right.$ $\left.T^{*} d_{m}\right\}$ and $\left\{d^{\prime}, d^{\prime \prime} \in \operatorname{ker}(T)^{\perp} \mid d^{\prime}=T^{*} d_{m}^{\prime}, d^{\prime \prime}=T^{*} d_{m}^{\prime \prime}, d_{m}^{\prime}+d_{m}^{\prime \prime}=d_{m}\right\}$ coincide. Therefore,

$$
S^{T A \cap T B}\left(d_{m}\right)=\inf _{\substack{d^{\prime}+d^{\prime \prime}=T^{*} d_{m} \\ d^{\prime}, d^{\prime \prime} \in \operatorname{ker}(T)^{\perp}}}\left(S^{A}\left(d^{\prime}\right)+S^{B}\left(d^{\prime \prime}\right)\right) .
$$

At the same time, we have

$$
S^{T(A \cap B)}\left(d_{m}\right)=S^{A \cap B}\left(T^{*} d_{m}\right)=\inf _{\substack{d^{\prime}+d^{\prime \prime}=T^{*} d_{m} \\ d^{\prime}, d^{\prime \prime} \in \mathbb{R}^{n}}}\left(S^{A}\left(d^{\prime}\right)+S^{B}\left(d^{\prime \prime}\right)\right) .
$$

Finally, as image $\left(T^{*}\right)=k e r(T)^{\perp}$, we can conclude that for any $d \in k e r(T)^{\perp}$

$$
\inf _{\substack{d^{\prime}+d^{\prime \prime}=d \\ d^{\prime}, d^{\prime \prime} \in \mathbb{R}^{n}}}\left(S^{A}\left(d^{\prime}\right)+S^{B}\left(d^{\prime \prime}\right)\right)=\inf _{\substack{d^{\prime}+d^{\prime \prime}=d \\ d^{\prime}, d^{\prime \prime} \in \operatorname{ker}(T) \perp}}\left(S^{A}\left(d^{\prime}\right)+S^{B}\left(d^{\prime \prime}\right)\right)
$$




\section{References}

Aumann, R. J., and Hart, S. (1986). Bi-convexity and bi-martingales. Israel Journal of Mathematics, 54(2), 159-180.

Axler, S. J. (1997). Linear Algebra Done Right. New York: Springer.

Gershkov, A., Goeree, J. K., Kushnir, A., Moldovanu, B., and Shi, X. (2013). On the Equivalence of Bayesian and Dominant Strategy Implementation. Econometrica, 81, 197-220.

Goeree, J. K. and Kushnir, A. (2017). A Geometric Approach to Mechanism Design. Working Paper, University of New South Wales and Tepper School of Business, Carnegie Mellon University.

Hiriart-Urruty, J. B. and Lemaréchal, C. (2012). Fundamentals of Convex Analysis. Springer Science \& Business Media.

Laffont, J-J. and Maskin, E. (1980). A Differential Approach to Dominant Strategy Mechanisms. Econometrica, 48, 1507-1520.

Manelli, A. M. and Vincent, D. R. (2010). Bayesian and Dominant-Strategy Implementation in the Independent Private Values Model. Econometrica, 78, 1905-1938.

Milgrom, P. R. (2004). Putting Auction Theory to Work. Cambridge University Press.

Myerson, R. (1979). Incentive Compatibility and the Bargaining Problem. Econometrica 47, 61-73.

Rockafellar, R. T. (1997). Convex Analysis. Princeton University Press.

Roth, A. E. (2008). What Have We Learned From Market Design?. The Economic Journal, 118(527), 285-310. 